\title{
Segmentation of Carpal Bones from 3D CT Images Using Skeletally Coupled Deformable Models
}

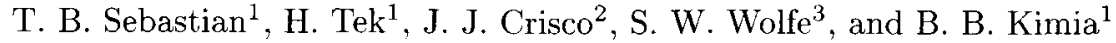 \\ 1 LEMS, Division of Engineering, Brown University, Providence RI 02912 \\ \{tbs, tek, kimia\}@lems . brown.edu, http://www. lems.brown.edu \\ 2 Department of Orthopaedics, Rhode Island Hospital, Providence, RI 02903 \\ Joseph_Crisco_IIIQBrown.edu \\ 3 Department of Orthopaedics, Yale University School of Medicine \\ Scott.Wolfe@yale.edu
}

\begin{abstract}
The in vivo investigation of joint kinematics in normal and injured wrist requires the segmentation of carpal bones from 3D (CT) images and their registration over time. The non-uniformity of bone tissue, ranging from dense cortical bone to textured spongy bone, the irregular, small shape of closely packed carpal bones which move with respect to one another, and with respect to $\mathrm{CT}$ resolution. augmented with the presence of blood vessels, and the inherent blurring of CT imaging renders the segmentation of carpal bones a challenging task. Specifically, four characteristic difficulties are prominent: (i) gaps or weak edges in the carpal bone surfaces, (ii) diffused edges, (iii) textured regions, and, (iv) extremely narrow inter-bone regions. We review the performance of statistical classification, deformable models, region growing, and morphological operations for this application. We then propose a model which combines several of these approaches in a single framework. Specifically, initialized seeds grow in a curve evolution implementation of active contours, but where growth is modulated by a skeletally-mediated competition between neighboring regions, thus combining the advantages of local and global region growing methods, region competition and active contours. This approach effectively deals with many of the difficulties presented above as illustrated by numerous examples.
\end{abstract}

Acknowledgements: We gratefully acknowledge the support of the Whitaker Foundation, and NIH grant AR44005.

\section{Introduction}

Segmentation is an important pre-processing step in medical imaging for visualization, registration, kinematic analysis, etc. For our application, namely, the segmentation and registration of carpal bones in the wrist from CT images, which is used primarily to investigate joint loading and joint kinematics, the task has proved to be rather challenging. A key fact is that bone tissue cannot 

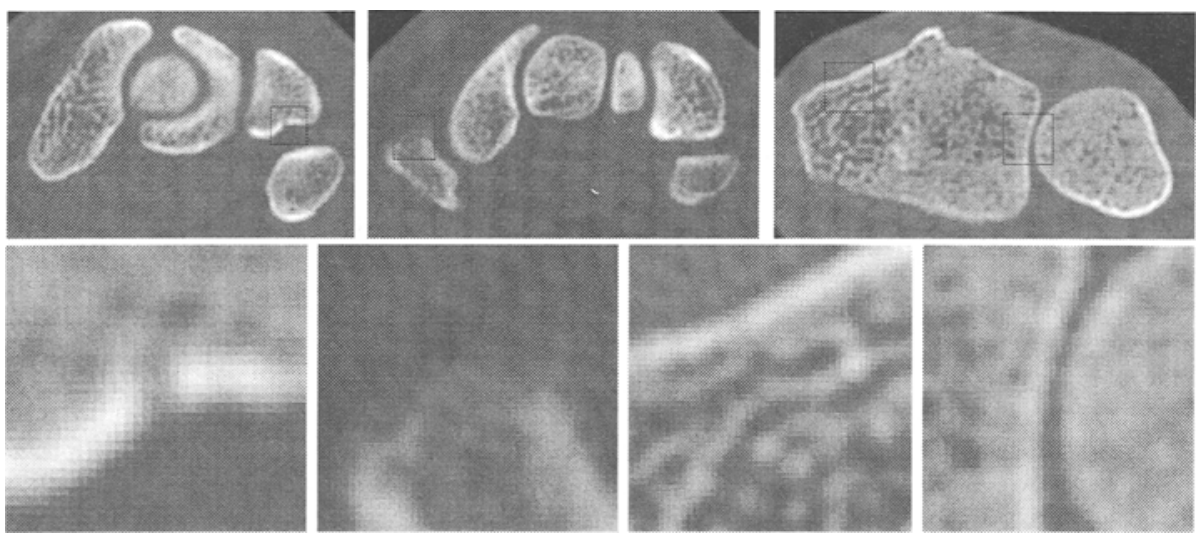

Fig. 1. Three slices from a three-dimensional CT image of carpal bones (top row) demonstrate typical cases of gaps or weak edges, diffused edges, textured areas, and extremely narrow inter-bone regions that make automatic segmentation difficult. Each window is zoomed in the bottom row detailing the above features, respectively.

be characterized uniformly: the outer layer of the bone tissue, or cortical bone, is denser than the spongy bone it encases. Thus, under CT imaging cortical bone appears brighter and smooth, while spongy bone appears darker and textured. In addition, due to the close spacing of some carpal bones with respect to CT resolution and inherent blurring in CT imaging, the inter-bone space often appears brighter than the background (soft tissue), drastically reducing boundary contrast in these regions. Finally, blood vessels resemble the background, creating gaps in the surface of bone images. In the image domain, challenging areas can be categorized into four predominant groups, Figure 1: (i) gaps in the cortical shell; (ii) weak or diffused bone boundaries due to partial volume effect in CT imaging; (iii) textured areas corresponding to the spongy bone; (iv) the narrow inter-bone regions which tend to be diffused.

While is possible for medical experts to segment these images by using thresholding or manual seeding, e.g. using the ANALYZE package, this process considering the time required for manual correction is labor intensive. Thus the development and use of segmentation techniques that minimize user interaction is highly desirable. We have implemented and evaluated a large number of techniques for the segmentation of carpal bones from 3D CT images, including global thresholding, statistical methods, seeded region growing [1], deformable models like snakes [7], balloons [4], and bubbles [18], morphological watersheds [20], and region competition [21]. The experience with the use of these techniques in our domain has prompted us to combine three of these approaches in a single framework. Specifically, this framework evolves from the approach taken by Tek et al. [18], numerous seeds are initialized which then grow by image-dependent forces and in the process merge and slow down near boundaries, thus trapping the boundary between the inner and outer regions. The success of this technique is dependent on the existence of boundaries with sufficient contrast or symmetric initialization in the case of weak boundaries. This is due to the monotonic nature 
of growth: once a region has evolved beyond object boundaries it can no longer return to capture it. Region competition [21], on the other hand, also relies on the growth of seeds, but implements a non-monotonic adjustment based on local competition between regions, once they become adjacent. The back and forth movements of adjacent regions is dependent on a statistical decision on which of these regions a point is more likely to belong to. The central assumption underlying this scheme is that the growth of seeds leads to regions that characterize distinct areas. This assumption fails, e.g. in asymmetrically initialized seeds, where the growing seeds in "waiting" for other regions to arrive, acquire and encompass two statistically distinct domains, thus disabling a reversal. Seeded region growing [1] avoids this difficulty by implementing a global competition among growing regions, but does not implement the "back and forth" competition between them, thus not allowing for recovery from errors.

The approach presented in this paper combines these ideas, namely, deformable models implemented in the curve evolution framework, probabilistic growth, local back and forth competition, and global competition under one framework. The main idea is to rely on the inter-region skeleton as a predictor of boundaries resulting from the current seeds, assuming current growth conditions, and to feed back this information into the growth process by modulating the deformation speed. In other words, if points on the skeleton are more likely to belong to one region as compared to another, then the former region should grow faster to capture it. Region competition then becomes a special case, i.e., when the two regions become adjacent. The idea of global competition in seeded region growing is implemented by the long-distance competition among neighboring seeds, mediated by the inter-region skeleton.

\section{Medical Application: Segmentation of Carpal Bones}

Degenerative joint disease is commonly attributed to alterations in joint loading and joint kinematics due to traumatic injury. In the wrist, despite widespread clinical awareness of dynamic and static wrist instability, little is known about the pathoanatomy and kinematics of these conditions. Patients may continue to be incapacitated by pain following stressful activities months after injury, even though radiographs and other static imaging studies appear normal. Attempts to treat these conditions surgically usually involve limiting abnormal carpal bone motion by restricting normal carpal motion through arthrodesis or ligamentous reinforcement. Characterizing the true 3D kinematics of the carpal bones following these ligament injuries would provide better insight for development of diagnostic techniques and more appropriate treatment strategies. Clarifying the relationship of partial and complete tears of the scapholunate interosseous ligament, for example, to alterations in carpal kinematics would help guide clinicians in decisions regarding surgical or conservative management of this common injury.

The significance of accurately measuring $3 \mathrm{D}$ in vivo carpal kinematics in normal wrists and in those with specific ligament injuries is the understanding 

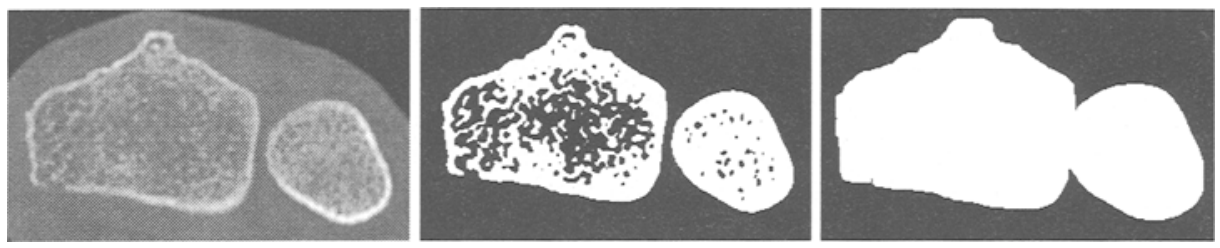

Fig. 2. An example of a $2 \mathrm{D}$ slice where global thresholding fails. Observe that there are holes in the bones and also a gap in the bone contour. While morphological filtering can close the holes and gaps, the inter-bone region will also be closed and the shape of the boundary will be slightly altered.

gained from these studies can benefit diagnosis, surgical treatment, rehabilitation, and the design of prosthetic devices. Our long-term research is aimed at studying degenerative changes, surgical reconstructions, and rehabilitation in joints. More broadly, these methods can noninvasively measure 3D motion of any joint using any $3 \mathrm{D}$ imaging modality.

Studying 3D skeletal joint motion is most often accomplished using marker systems implanted in the bones of cadaveric specimens. Similar methods have also been used to study joint motion in vivo in the knee and hip $[6,10,11]$, but these studies are not widely performed due to their invasive nature. More recently, researchers have measured the $3 \mathrm{D}$ kinematics of the knee joint in vivo using non-invasive techniques based on single-plane fluoroscopy and three dimensional models [2]. However, given the small size, narrow articular spaces and complex joint surfaces, such methods are not applicable to the wrist. We now review an application of some of the currently available techniques to this domain, and then proceed by an approach that combines some of the current techniques.

\section{Segmentation of Carpal Bones: Current Approaches}

We have investigated the use of several segmentation techniques for the recovery of carpal bone surfaces from $\mathrm{CT}$ images. These methods include global thresholding, statistical classification, seeded region growing [1], region competition [21], deformable models like snakes [7], balloons [4], their curve evolution counterparts $[12,3,17]$, and watershed segmentation [20]. See also [16] for a similar application. This section briefly reviews our experience with the application of these techniques to carpal bone segmentation and motivates the approach present in Section 4.

Global thresholding is the simplest statistical segmentation technique, where pixels are classified based on their intensity values. However, choosing the right intensity threshold is difficult and typically varies from one dataset to another. Interactive manual selection of the threshold is tedious and operator-sensitive. Even with the optimal threshold, final segmentation based on thresholding has holes and in some images, two adjacent bones merge. The choice of the intensity threshold can be automated by using the expectation maximization (EM) algorithm [5] to fit a mixture of Gaussian distributions to the intensity values and using classical Bayes Decision theory to find the decision boundaries. 

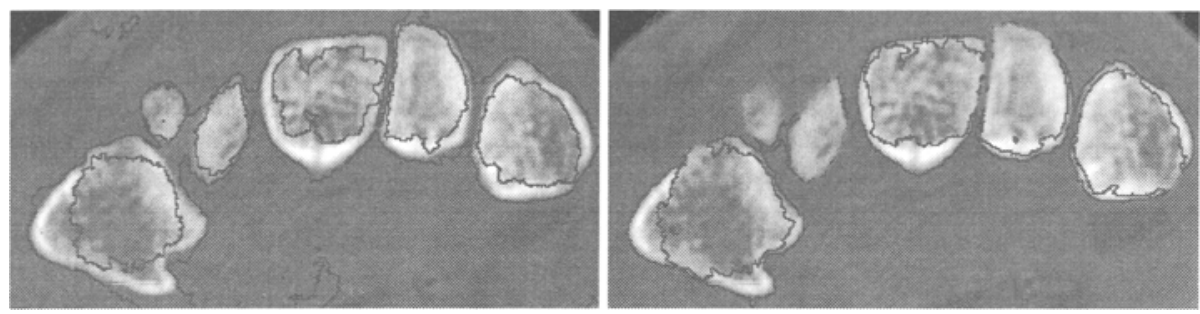

Fig. 3. This figure illustrates a shortcoming of the bubbles [17] approach. (Left) Randomly initialized seeds have grown to near bone boundaries; note the convergence of bubbles in the small bone on the top left. (Right) Further iterations, which are necessary for convergence in other places, e.g., inter-bone regions will push out the bubble inside the small bone.
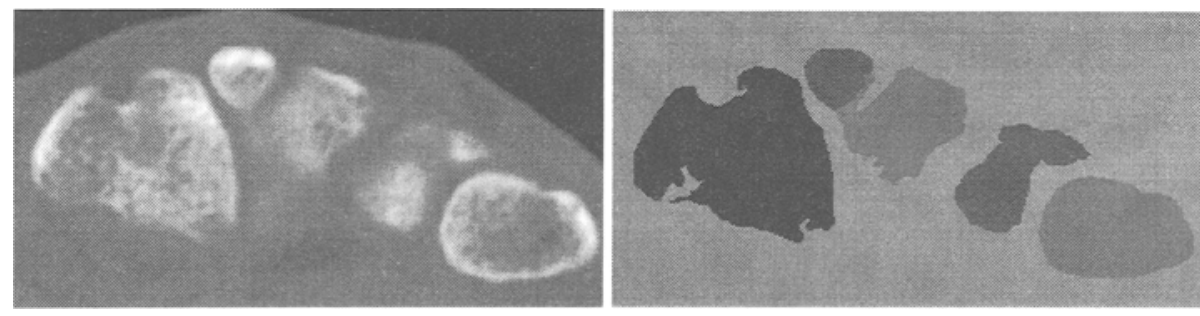

Fig. 4. This figure illustrates a shortcoming of seeded region growing [1]. Seeded region growing can leak through gaps in the bones.

Deformable models (snakes or active contours [7]) need to be initialized close to the boundaries for proper convergence, one snake per boundary. Balloons overcome the first restriction by transporting the initialized model close to the edges by adding a constant inflation force to the snake [4]. However, this inflation term also often pushes the evolving contour over weak/diffused edges. Curve evolution snake models $[12,3,17]$ address the second restriction since they have the ability to change their topology during the deformation process. However, for carpal bone segmentation, the deformable models encounter similar problems, as in using statistical classification: (i) not all models converge at weak/diffused boundaries; (ii) the contour smoothing terms does not allow entry into the narrow inter-bone region; (iii) as these models rely only on the local information along the boundary, the texture inside the bone slows snakes down, resulting in poor convergence on the bone boundary.

Another class of techniques for segmentation is region growing and merging, where initialized seeds grow by annexing "similar" (as defined by a statistical test) pixels. Region growing methods are sensitive to seed initialization, and result in jagged boundaries and poorly localized edges. Seeded region growing [1] improves traditional region growing by introducing a "competition" between growing regions by ordering all candidate "growth" pixels according to some suitability criteria. However, seeded region growing, like traditional region growing doesn't incorporate any geometrical information and hence can "leak" through 

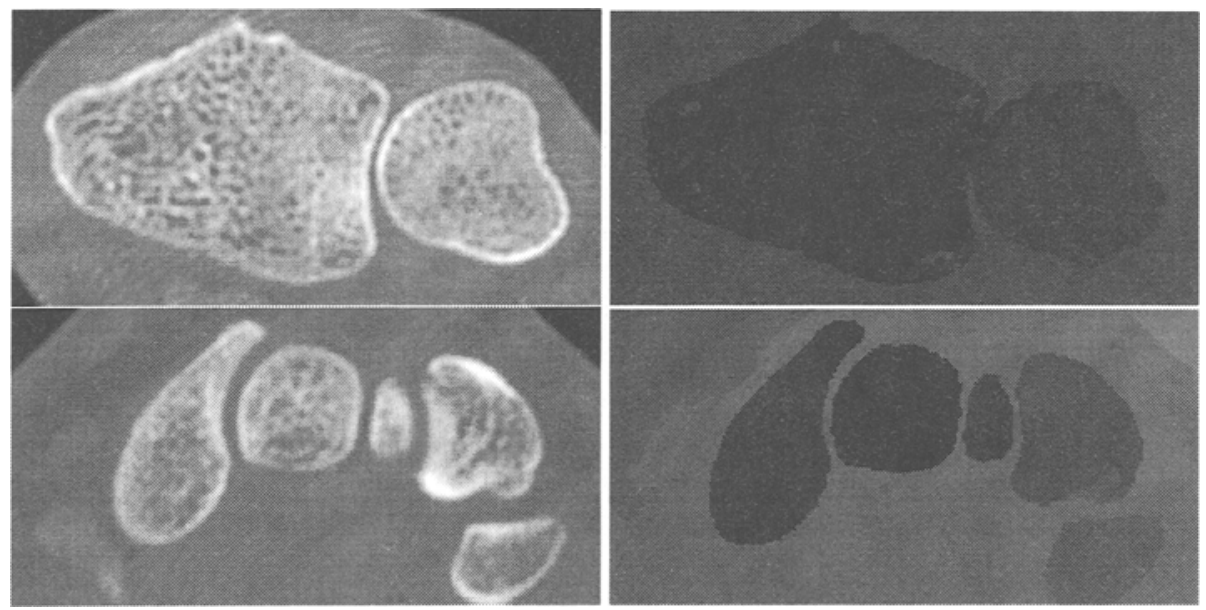

Fig. 5. While region competition [21] frequently captures the correct bone boundaries: in some cases it does not, as illustrated here. In the first case, region competition merges the bones and there are some holes in the captured regions. In the second case region competition fails to segment the bone on the extreme left of the image. Here the seeds that were initialized lost statistical character, resulting in the wrong segmentation, a mislabelling of some bones. The main flaw is that seeds compete only after they have fully grown and become adjacent.

narrow gaps (like the ones seen in the cortical shell, Figure 1). It also tends to merge bones that are very close to each other. Region competition [21] combines the geometrical features of the deformable models and the statistical features of region growing, by using a combination of statistical and smoothing forces for seed growth. It also introduces a local competition between regions when they contact each other, thus allowing recovery from errors. This is a powerful technique that works even when seeds are initialized across boundaries. However, for carpal bone segmentation it was observed that occasionally, initialized regions which have grown to a great extent beyond object boundaries, lose their "character" and hence are unable to be pushed back. Also, the discrete nature of region competition allows the boundaries to move by one pixel or not at all, resulting in jagged boundaries.

Finally, watershed segmentation [20] can be viewed as a region-growing technique [18] which often results in over-segmentation, unless specialized marker methods [20] are used. See [13] for details.

\section{Skeletally Coupled Deformable Models}

Our proposed approach is an iterative one where initialized seeds grow by a local statistical force, modulated by the desirability of a simulated segmentation, assuming current conditions prevail. The main idea is (i) at each step a local growth force is computed, (ii) the skeleton of the inter-seed region is used as a predictor of the final segmentation, (iii) each skeleton point is viewed as the 

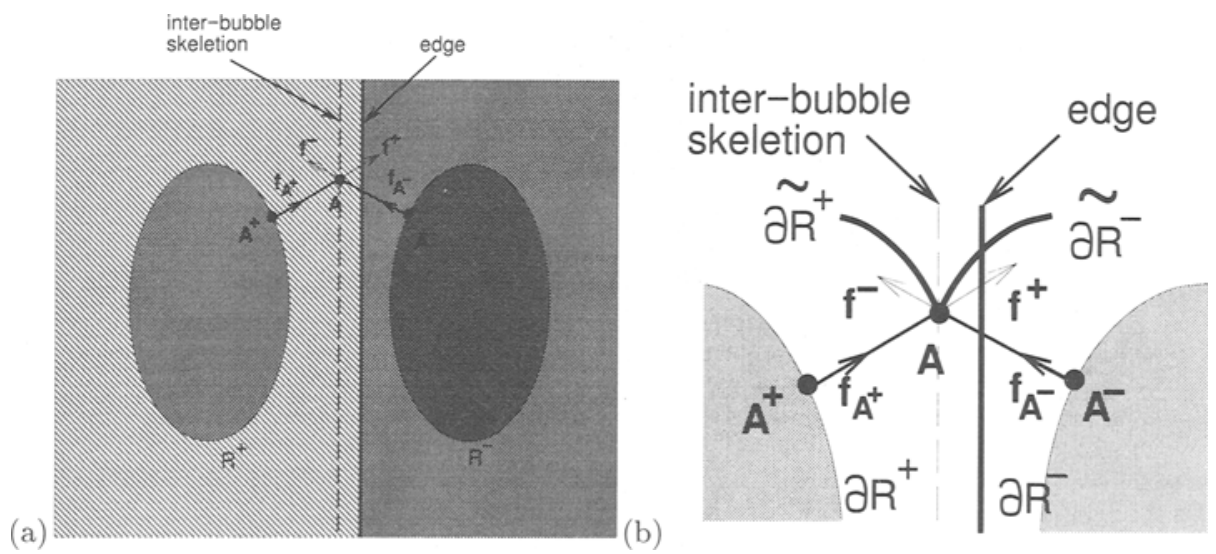

Fig. 6. (a) A sketch of the coupling of forces between nearby regions, (seeds of bubbles) $R^{+}$and $R^{-}$, e.g., coupling of $A^{+}$and $A^{-}$through $A$. (b) Actual coupling in a cropped portion; the thin solid lines are the boundaries of each each deformable model, $R^{+}$and $R^{-}$, the thick solid line is the deformable true boundary (edge), and the dashed line is the inter-bubble skeleton. The skeleton is a preview of the final segmentation should the current growth conditions prevail. The relative desirability of growth from $A^{+}$to $A$ as compared to that of $A^{-}$to $A$ modulates the growth of $A^{+}$and $A^{-}$in each iteration, in this case inhibiting the growth of $A^{-}$and encouraging the speedier growth of $A^{+}$. Hence the skeleton, recomputed in each iteration converges onto the edge.

front of competition for a pair of points, each from a different seed, (iv) the desirability of growth of each seed point, along its respective paths to form the skeleton, is statistically measured and compared, and (v) the relative merit of each path is used to modulate the local growth force at each seed point to improve the segmentation results. This approach, namely, skeletally coupled deformable models (SCDM) thus implements a "long range coupling" that allows for the competition at a distance and prevents the seeds from losing their statistical characterization when seeds are initialized close to or over the boundary.

To see how long range coupling is mediated by the inter-region skeleton, consider Figure 6, which illustrates two seeds $R^{+}$and $R^{-}$and the background $R_{B}$. Let $\mathcal{S}$ denote the shocks ${ }^{1}$ of $R_{B}$ as defined in $[8,9,14,19]$. Each shock point $A$ couples two ${ }^{2}$ seed points $A^{+} \in \partial R^{+}$and $A^{-} \in \partial R^{-}$, where $\partial R$ is the boundary of $R$. This coupling of the deformable models' boundary points, $A^{+}$ and $A^{-}$, allows for competition for their growth with respect to capturing $A$. Let the local statistical growth force at a point $P$ be denoted by $f_{P}$, typically $f_{P}=\log \left(\frac{1}{\sqrt{2 \pi}} \overline{\sigma^{2}} e^{-\frac{(I(x, y)-\mu)^{2}}{2 \sigma^{2}}}\right)$, where $I(x, y)$ is the intensity at $P$ and $\mu$ and $\sigma$ are the mean and standard deviation of the region. This defines local forces $f_{A^{-}}$

${ }^{1}$ Shocks are the skeleton points augmented with the notions of speed, direction, type, label, grouping, and a hierarchy of these groups [9].

${ }^{2}$ Generically, each shock point couple two or three boundary (characteristic) points. The discussion for shocks with three characteristic points is similar. 
and $f_{A^{+}}$, at $A^{-}$and $A^{+}$, respectively,

$$
f_{A^{-}}=\log \left(\frac{1}{\sqrt{2 \pi \sigma^{-2}}} e^{-\frac{\left(I(x, y)-\mu^{-}\right)^{2}}{2 \sigma^{-2}}}\right), \quad f_{A^{+}}=\log \left(\frac{1}{\sqrt{2 \pi \sigma^{+^{2}}}} e^{-\frac{\left(I(x, y)-\mu^{+}\right)^{2}}{2 \sigma^{+2}}}\right) .
$$

These forces are adjusted to produce net forces, $F_{A^{-}}$and $F_{+}$, respectively by using feedback indicating the desirability of a predicted segmentation at $A$, assuming $A^{-}$and $A^{+}$evolve in a simulated growth up to collision at A. Since only infinitesimal adjustments are needed, we consider whether it is more appropriate for the skeleton to move left or right, and by what amount. This is accomplished by comparing the predicted statistical forces $f^{+}$and $f^{-}$at $A$.

$$
f^{+}=\log P\left(A \in R^{+}\right), \quad f^{-}=\log P\left(A \in R^{-}\right) .
$$

Thus, the net force at each skeletal point $A, f^{+}-f^{-}$, is propagated to each boundary point to modulate the growth force there. This force is only needed when boundaries are at a distance. Second, in addition to the local skeleton force, we feed back a measure of growth at $A^{+}$to $A^{-}$(and vice-versa) via coupling at the skeleton, as adjusted by a monotonically decreasing function of the distance $\lambda(d)=\frac{1}{\sqrt{2 \pi \sigma_{d}^{2}}} e^{-\frac{d^{2}}{2 \sigma_{d}^{2}}}$. Thus, the net force is

$$
\begin{aligned}
& F_{A^{-}}=f_{A^{-}}-\lambda_{0} f_{A^{-}}+\left(1-\lambda_{0}\right)\left(f^{-}-f^{+}\right) \\
& F_{A^{+}}=f_{A^{+}}-\lambda_{0} f_{A^{+}}+\left(1-\lambda_{0}\right)\left(f^{+}-f^{-}\right)
\end{aligned}
$$

where $\lambda_{0}=\lambda\left(2 d\left(A^{-}, A\right)\right)$. When two regions are close to each other, or adjacent, $\lambda_{0} \approx 1, F_{A^{-}}=f_{A^{-}}-f_{A^{+}}$, effectively implementing the region competition approach. However, when the two regions are not close, the approach implements a long range, predicted competition. In Figure 6, this long range force has the effect of slowing $A^{-}$down and speeding up $A^{+}$, allowing $A^{+}$to "catch up" before $A^{-}$has grown beyond the edge, thus symmetrizing the regions $R^{+}$and $R^{-}$with respect to the edge.

\section{Implementation: Curve Evolution and ENO Interpolation}

This approach is implemented in the curve evolution framework by embedding the curve as the zero level-set of an evolving surface. This allows for partial movement of the curve on a discrete grid. Partial movements are essential to avoid jagged boundaries and to allow regions to move at different speeds. However, a subpixel implementation of region competition requires regions to be adjacent, thus constantly operating in a mode where more than one curve is present within a pixel, a situation not easily represented by an embedding surface. Specifically, the reliable identification of the subpixel boundary and the computation of forces at subcell points are especially challenging. 
(a)

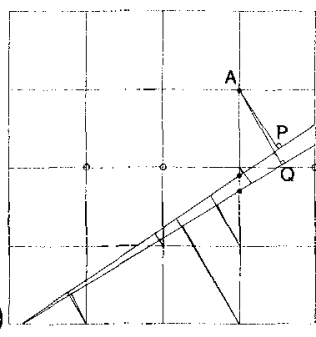

(b)

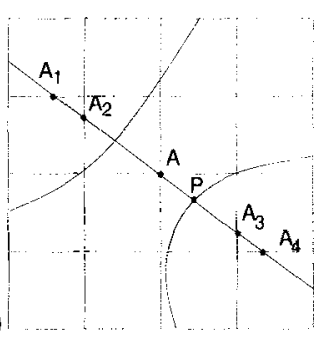

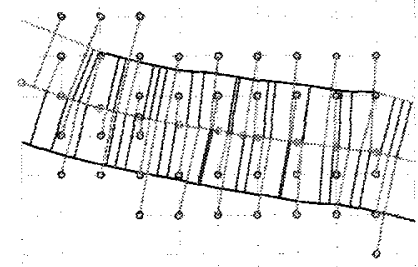

(c)

Fig. 7. The subpixel deformation of a curve via updating its embedding surface by the movement of the closest point to each grid point. (a) The subpixel computation of force at $\mathrm{P}$ requires a two stage ENO interpolation; one along the line normal to the curve $\left\{A_{i}\right\}$ and one for each $A_{i}$ along either the horizontal or vertical directions (solid dot). (c) The coupling between two contours $\partial R^{+}$and $\partial R^{-}$when they are roughly two pixels apart. Details are omitted here due to space constraints.

As a solution, we use a piecewise circular (PC) approximation [15], of the subpixel boundary within each pixel. This representation can be extracted accurately from its distance transform using an approach based on Essentially Non Oscillatory (ENO) interpolation [15]. The accuracy of the PC curve reconstruction from its embedding surface, implies that the surface can be transformed in such a way that the modified surface is the distance transform of the modified curve. Hence, the surface point at $A$ in Figure 7(a) should be updated by the change from $A P$ to $A Q$, which for small movements can be approximated by $P Q$. This requires computing the force at subcell point point $P$, which requires the accurate computation of the image intensity and image derivatives along the normal to the curve at $P$. This is done using an ENO interpolation along the line orthogonal to the curve, along the subpixel grid samples $\left\{A_{i}\right\}$ in Figure $7(\mathrm{~b})$, which in turn is found using an ENO interpolation along grid lines. The details of the implementation are involved and beyond the scope of this paper [13].

\section{Results and Discussion}

In this section we present the results of applying the SCDM method to carpal bone segmentation, especially to illustrate the performance of SCDM in the problem areas listed in Section 1, namely, gaps and weak edges, diffused edges, bone texture, and narrow inter-bone spaces. Seeds were initialized manually, one seed per bone (this can be automated at a later stage by relying on statistical classification). Figure 8 illustrates the effectiveness of this approach in images with gaps (a), low contrast edges (b) and (c), narrow regions (d), and diffused edges (e). These are typical results and the application of this method to carpal bone images has led to reliable and robust segmentation. For further examples, animation of growth, and comparison to other techniques, we refer the reader to http://www.lems.brown.edu/vision/researchAreas/SCDM/bonesegmentation/bone-segmentation.html. 


\section{References}

1. R. Adams and L. Bischof. Seeded region growing. PAMI, 16(6):641-647, 1994.

2. S. A. Banks and W. A. Hodge. Accurate measurement of three-dimensional knee replacement kinematics using single-plane fluorscopy. IT Biomed Eng, 43(6):638649, 1996.

3. V. Caselles, F. Catte, T. Coll, and F. Dibos. A geometric model for active contours in image processing. Numerische Mathematik, 66, 1993.

4. L. D. Cohen and I. Cohen. Finite element methods for active contour models and balloons for 2D and 3D images. PAMI, 15:1131-1147, 1993.

5. A. Dempster, N. Laird, and D. Rubin. Maximum likelihood from incomplete data via the EM algorithm. J. of Royal Stat. Soc., Series B, 39:1-38, 1977.

6. J. Karrholm, L. Elmqvist, G. Selvik, and L. Hansson. Chronic antrolateral instability of the knee: a Roentgen stereophotogrammetric evaluation. Am. J. Sports Medicine, 17(4):555-563, 1989.

7. M. Kass, A. Witkin, and D. Terzopoulos. Snakes: Active contour models. IJCV, $1: 321-331,1988$.

8. B. B. Kimia, A. R. Tannenbaum, and S. W. Zucker. Toward a computational theory of shape: An overview. In ECCV '90, pages 402-407, 1990.

9. B. B. Kimia, A. R. Tannenbaum, and S. W. Zucker. Shapes, shocks, and deformations, I: The components of shape and the reaction-diffusion space. $I J C V$, 15:189-224, 1995.

10. T. J. Koh, M. Grabiner, and R. DeSwart. In vivo tracking of the human patella. J. Biomech., 25(6):637-644, 1992.

11. M. Lafortune, P. Cavanagh, H. Sommer, and A. Kalenak. Three-dimensional kinematics of the human knee during walking. J. Biomech., 25(4):347-358, 1992.

12. R. Malladi, J. A. Sethian, and B. C. Vemuri. Evolutionary fronts for topologyindependent shape modelling and recovery. In ECCV '94, pages 3-13, 1994.

13. T. B. Sebastian, H. Tek, J. J. Crisco, and B. B. Kimia. Skeletally coupled deformable models. Tech. Rep. LEMS 169, LEMS, Brown University, January 1998.

14. K. Siddiqi and B. B. Kimia. A shock grammar for recognition. In CVPR '96:507$513,1996$.

15. K. Siddiqi, B. B. Kimia, and C. Shu. Geometric shock-capturing ENO schemes for subpixel interpolation, computation and curve evolution. GMIP, 59(5):278-301, 1997.

16. H. D. Tagare and K. W. Elder. Location and geometric descriptions of carpal bones in CT images. Annals of Biomedical Engineering, 21:715-726, 1993.

17. H. Tek and B. B. Kimia. Image segmentation by reaction-diffusion bubbles. In ICCV, pages 156-162, Boston, June 1995.

18. H. Tek and B. B. Kimia. Volumetric segmentation of medical images by threedimensional bubbles. CVIU, 64(2):246-258, 1997.

19. H. Tek, F. Leymarie, and B. B. Kimia. Multiple generation shock detection and labeling using CEDT. In $I W V F$ '97, Italy, May 1997.

20. L. Vincent and P. Souille. Watersheds in digital spaces: an efficient algoritm based on immersion simulations. PAMI, 13(6):583-598, 1991.

21. S. C. Zhu and A. L. Yuille. Region competition: Unifying Snakes, Region growing, and Bayes/MDL for multiband Image Segmentation. PAMI, 18(9):884-900, 1996. 
(a)
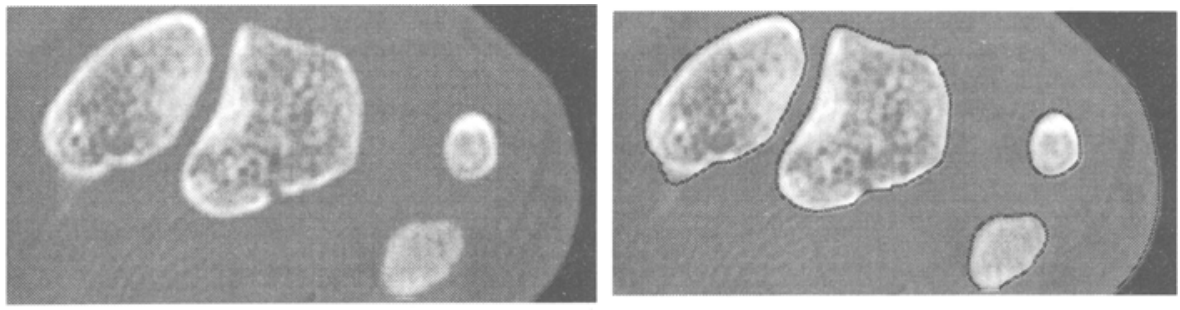

(b)
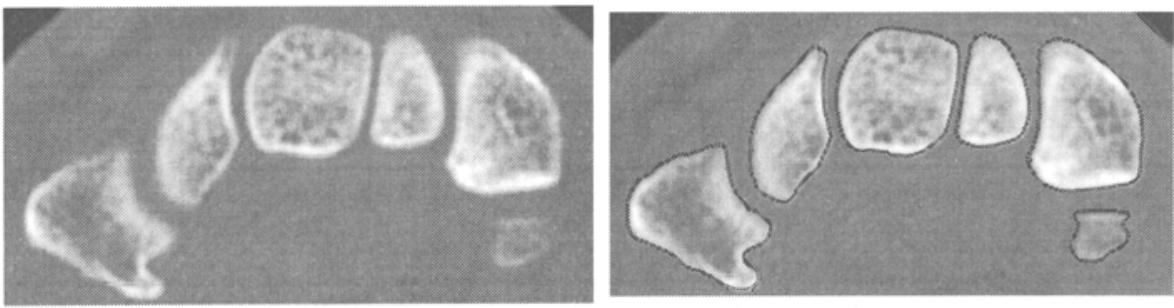

(c)
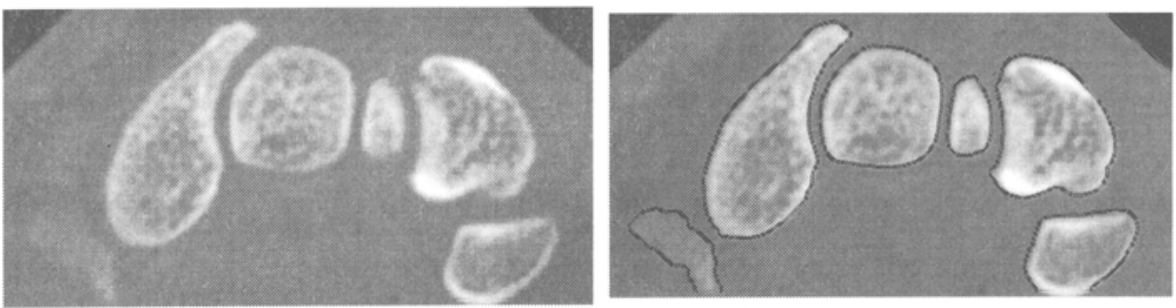

(d)
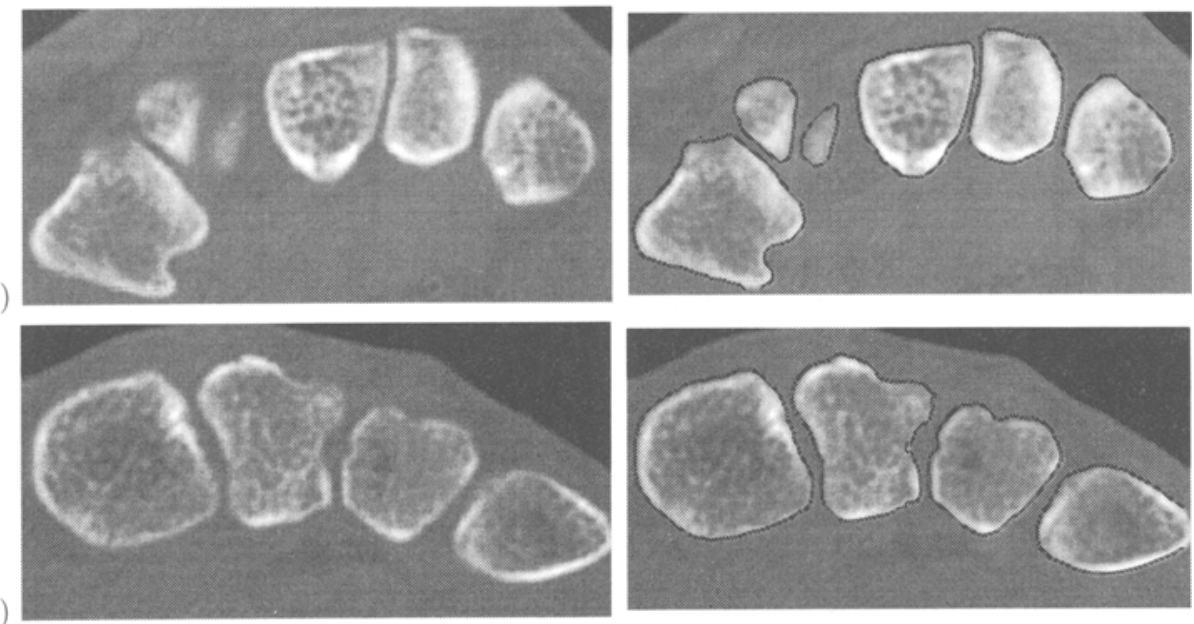

Fig. 8. Examples of carpal bone segmentation using skeletally coupled deformable models. Observe that SCDM works well in images having a gap in the bone contour, (a). It captures low contrast contours, e.g., the bone on the bottom right in (b) and the bone on the extreme left in (c). Observe how it allows growth into the narrow inter-bone spaces, (d). It also does well in case of diffused edges, e.g., second bone in (e). Also note that the region-based statistical force allows growth in textured areas, as evidenced by all these examples. 